\title{
Post-encoding reactivation promotes one-shot learning of episodes in humans
}

Xiongbo $\mathrm{Wu}^{1-3}$, Xavier Viñals ${ }^{4}$, Aya Ben-Yakov ${ }^{5}$, Bernhard P. Staresina ${ }^{6}$, Lluís Fuentemilla $a^{1-3}$

${ }^{1}$ Cognition and Brain Plasticity Group, Bellvitge Institute for Biomedical Research, Hospitalet de Llobregat 08907, Spain; ${ }^{2}$ Department of Cognition, Development and Educational Psychology, University of Barcelona, Barcelona 08035, Spain; ${ }^{3}$ Institute of Neurosciences, University of Barcelona, Barcelona 08035, Spain. ${ }^{4}$ High School of Health Science, Tecnocampus Pompeu Fabra University, 08302 Mataró (Barcelona), Spain; ${ }^{5}$ Medical Research Council Cognition and Brain Sciences Unit, University of Cambridge, Cambridge CB2 7EF, United Kingdom; ${ }^{6}$ School of Psychology and Centre for Human Brain Health, University of Birmingham, UK

Corresponding author: Lluís Fuentemilla. Department of Cognition, Development and Educational Psychology, University of Barcelona. Feixa Llarga s/n, 08907, L'Hospitalet (Barcelona), Spain Phone: +34-(0) 934021038 Fax: +34-(0) 4024268; Email: Ilfuentemilla@ub.edu 


\begin{abstract}
Much work in rodents and in humans has provided evidence that post-encoding reinstatement plays an important role in stabilizing memory beyond initial learning processes. However, it remains unclear whether memory reinstatement is important for the rapid - 'oneshot' - learning of an unfolding episode. Here, we asked whether the reinstatement of an episode may occur preferentially post-encoding, when an individual perceives a meaningful event to be concluded. We asked human participants (male and female) to encode sequences of pictures depicting unique episodic-like events. We used representational similarity analysis of scalp electroencephalography recordings during encoding and found evidence for memory reactivation of the just encoded sequence of elements at the offset of the episode. Importantly, memory reinstatement was not observed between successive elements within an episode, indicating memory reactivation was specifically induced once participants perceived the unfolding episode to be completed. We also found that memory reinstatement predicted memory recollection of an encoded episode and that offset memory reinstatement was not present when participants encoded sequences of pictures that were not perceived as meaningful episodes. These results indicate that memory reinstatement at episode offsets is a mechanism selectively engaged to support rapid memory formation of single events.
\end{abstract}




\section{Introduction}

In episodic encoding, an experienced event is rapidly transformed into a memory trace that has the potential to be consciously recollected at long-term (Tulving, 1983). Prior research has largely focused on examining how the brain contributes to successful encoding of individual trial information, such as single images (Paller and Wagner, 2002) or single itemcontext associations (Davachi, 2006). However, in natural settings, an episode is better characterized by a collection of successive elements that become contextually meaningful as they unfold over time. In order to be accessible for future retrieval, these elements have to be associatively linked into a durable memory trace. Discerning "when" the brain binds the continuous experience input into a cohesive episodic memory trace and "how" the brain undergoes this rapid transformation is essential to understand memory formation.

Human neuroimaging studies examining memory formation during a continuous stream of stimuli, such as naturalistic video clips, have shown that a distributed network of brain regions comprising the hippocampus and neocortex increased activity at the end of an event (Ben-Yakov et al., 2013; Ben-Yakov and Henson, 2018; Baldassano et al., 2017). This event offset brain signal has been shown to reflect a binding operation of the just encoded event elements into a specific spatio-temporal context (Ritchey and Cooper, 2020), which aligns well with the notion that the hippocampus is crucial for binding elements of our experience with contextual information (Davachi, 2006; Diana et al., 2007; Eichenbaum et al., 2007; Ranganath, 2010). In real life, episodic encoding relies on the possibility to form a coherent memory trace that integrates the temporally evolving sequence of elements into a meaningful context, so that if there is a shift in contextual information this is perceived as the end of one episode and the beginning of another (Zacks et al., 2007). These episodic boundaries are thought to support the segmentation of the continuous experience into discrete episodes (Zacks et al., 2007) and their detection has a direct impact on how events are organized into meaningful units in long-term memory (Kurby and Zacks, 2008; Radvansky, 2012; Ezzyat and Davachi, 2011; DuBrow and Davachi, 2013, 2014).

Work in rodents has provided evidence that memory replay at event offset plays an important role in stabilizing memory beyond initial learning processes (Foster and Wilson, 2006; Diba and Buzsáki, 2007; Karlsson and Frank, 2008; Carr et al., 2011). In humans, event offset memory reinstatement has been shown to be induced by salient changes in stimulus features (Sols et al., 2019), goal states (Schuck and Niv, 2019) or prediction error (Sinclair and Barense, 2019). However, it remains unclear if memory reinstatement of an episode is endogenously induced when an individual perceives a meaningful event to be concluded, i.e., 
once the unfolding experience can be fully integrated as a memory representation that can be later recollected.

In the current study, we tested the hypothesis that memory reinstatement of an episode would preferentially occur rapidly post-encoding. We asked participants to encode sequences of pictures depicting unique episodic-like events followed by a delay period with no stimulus. We used representational similarity analysis of scalp electroencephalography (EEG) recordings during encoding and found evidence for memory reactivation of the just encoded sequence elements of the episode after encoding, i.e., at the offset of the episode. Importantly, memory reinstatement was not observed between successive elements within an episode, indicating memory reactivation was specifically induced once participants perceived the unfolding episode to be completed. We also found that memory reinstatement predicted memory recollection of an encoded episode and that offset memory reinstatement was not present when participants encoded sequences of pictures that were not perceived as meaningful episodes. These results indicate that episodic offset memory reinstatement is a mechanism selectively engaged to support rapid memory formation of single episodes.

\section{Materials and Methods}

\section{Participants}

Participants were native Spanish speakers who were recruited for pay (10€/h). All participants had normal or corrected-to-normal vision and reported no history of medical, neurological or psychiatric disorders. Twenty-five participants (17 females, age range 18-29) were recruited for Experiment 1. In addition, twenty-eight new participants (15 females, age range 19-35) were recruited for Experiment 2. Informed consent was obtained from participants in accordance with procedures approved by the Ethics Committee of the University of Barcelona.

\section{Experimental Procedure}

Both Experiments (Fig. 1) consisted of an encoding and a retrieval phase, separated by a 10-15 mins-break in the middle. Task timing and visual stimulus presentation were under the control of commercially available software e-Prime 2.0 (Psychology Software Tools).

The encoding phase of Experiment 1 included 100 different pictures, separated into sequences of 6 pictures each. Sequence unfolded in a life-like, coherent narrative. The order of pictures within each sequence was the same across participants but the order of the sequence was counterbalanced across participants. Each trial began with the presentation of text 'New Episode' for 2000ms, which marked the start of a new sequence. This was followed 
bioRxiv preprint doi: https://doi.org/10.1101/2021.04.13.439658; this version posted April 14, 2021. The copyright holder for this preprint (which was not certified by peer review) is the author/funder, who has granted bioRxiv a license to display the preprint in perpetuity. It is made available under aCC-BY-NC 4.0 International license.

by a fixation screen with a red asterisk lasting $2500 \mathrm{~ms}$. Each picture was then presented sequentially on a white screen for $2500 \mathrm{~ms}$ and followed by a $1500 \mathrm{~ms}$ black fixation cross. Immediately after the presentation of the last picture in each series, a blue asterisk was presented on the screen, indicating a post-episode offset period of $4000 \mathrm{~ms}$ during which participants were previously instructed to avoid rehearsing the just-encoded picture sequence. The asterisk remained visible on the screen during the offset period. Immediately after the offset period, participants were asked to provide a subjective rating of coherence for the just encoded sequence. a rating scale ranged from 1 to 4 , where 1 stood for 'not coherent' and 4 stood for 'very coherent'. The next trial began after a fixed time interval of $2000 \mathrm{~ms}$.

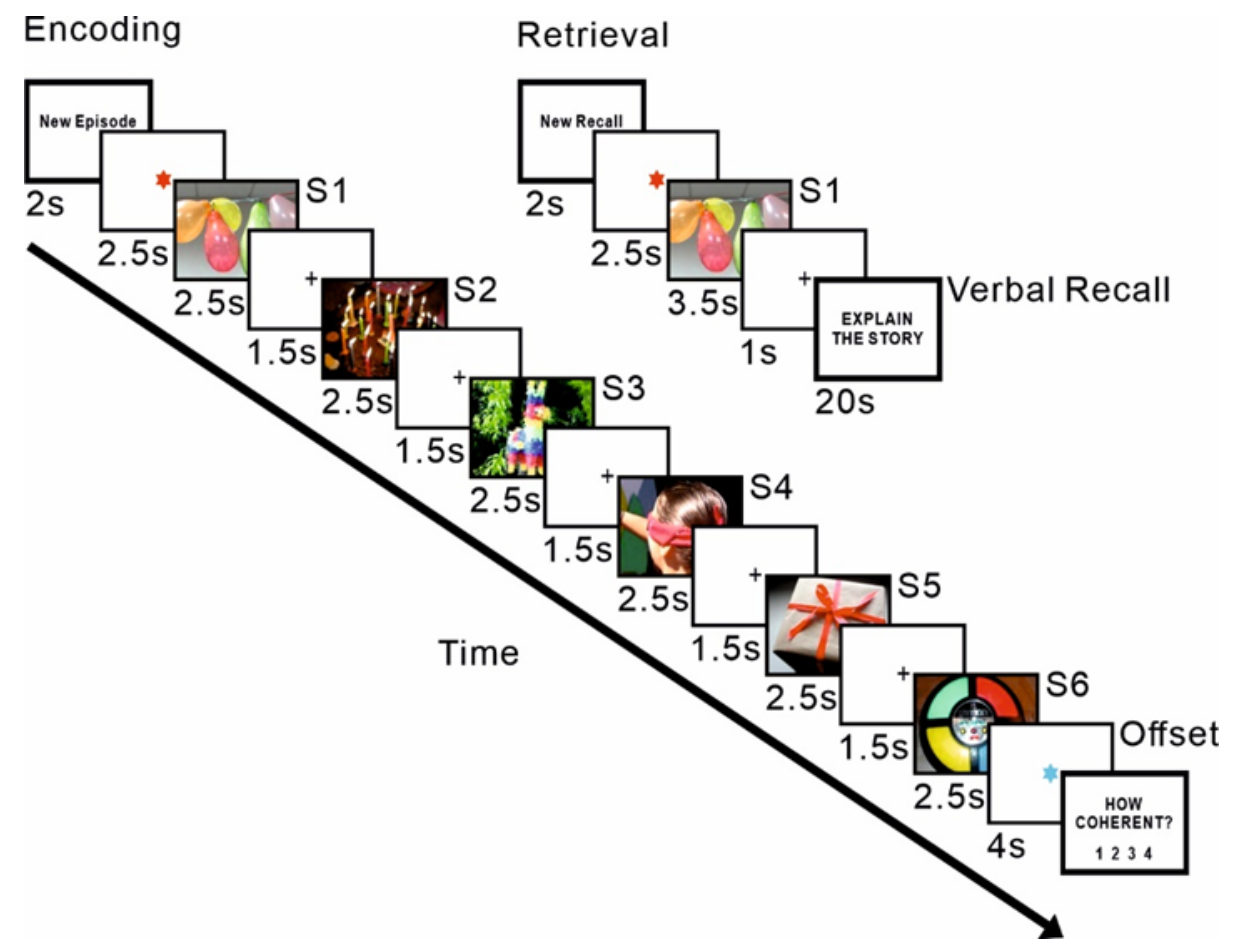

Figure 1. The experimental design in Experiment 1. During encoding, 100 different sequences were presented. Each sequence included 6 pictures that unfold a life-like coherent narrative episode. Each picture was presented $2.5 \mathrm{~s}$, followed by a $1.5 \mathrm{~s}$ fixation cross. After each sequence of images, there was an offset period (4s) during which participants were instructed to avoid rehearsing the just-encoded picture sequence. Participants were asked to provide a subjective rating of episodic coherence to the just encoded sequence at the end of each trial. Retrieval task was conducted 10-15 minutes after encoding. During retrieval, the first picture of each sequence was presented for $3.5 \mathrm{~s}$ which was followed fixation cross, and a message prompted at the screen instructing to report the associated episode during encoding. Participants were asked to verbally report within 20 seconds their memory associated episode or to indicate whether no memory came up associated to that picture cue.

For the retrieval phase, each trial started after the presentation of text 'New Recall' for $2000 \mathrm{~ms}$ on the screen. This was followed by a fixation screen with a red asterisk lasting $2500 \mathrm{~ms}$. After the asterisk, the first picture of one sequence was presented to participant for $3500 \mathrm{~ms}$ on the screen serving as a cue to prompt the free verbal recall for the rest of pictures in that sequence. Participants were instructed to start the verbal recall once the text 'Explain 
the story' was presented on the screen following a $1000 \mathrm{~ms}$ fixation cross. The verbal recall was limited to 20 seconds and participants could stop the recall when finished by pressing the space bar. The order of the picture cues was randomized before their presentation at the retrieval phase.

In Experiment 2, we shuffled the pictures across sequences so that each sequence was formed by 6 pictures from different sequences. Thus, each sequence no longer depicted a meaningful episodic sequence. Sixty shuffled sequences were selected for Experiment 2, and order of pictures within each sequence was kept the same across participants. The order of the sequence was randomized between participants. The general experimental settings for the encoding and retrieval phases were identical to Experiment 1. However, given the high difficulty of the task, two adjustments were made. First, the presentation time of each picture during encoding was increased to $3000 \mathrm{~ms}$. Second, participants were requested to perform a sequence order recognition task after each cued-picture recall task. During the sequence order recognition task, all 6 pictures from the same sequence (including the cue picture) were presented on the screen in random positions and participants were asked to type the order in which they appeared in the encoding phase. Participants had 30 seconds to type the order of the pictures and they could skip to the next trial when finished by pressing the space bar.

\section{EEG Recording}

For both Experiments, EEG was recorded using a 31-channel system at a sampling rate of $500 \mathrm{~Hz}$, using a BrainAmp amplifier and $\mathrm{Ag} / \mathrm{AgCl}$ electrodes mounted in an electrocap (EasyCap) located at 28 standard positions (Fp1/2, Fz, F7/8, F3/4, Fc1/2, Fc5/6, Cz, C3/4, T3/4, Cp1/2, Cp5/6, Pz, P3/4, T7/9, P7/8, O1/2, Oz) and at the left and right mastoids. An electrode placed at the lateral outer canthus of the right eye served as an online reference. EEG was re-referenced offline to the linked mastoids. Vertical eye movements were monitored with an electrode at the infraorbital ridge of the right eye. Electrode impedances were kept below $3 \mathrm{k} \Omega$. A band-pass filter $(0.1 \mathrm{~Hz}-20 \mathrm{~Hz})$ was implemented offline before the analysis.

\section{Verbal recall analysis}

During the retrieval phase of Experiment $1 \&$ 2, participants were asked to verbally recall as many pictures as possible within each sequence corresponding to the cue. Free verbal recall of each trial was recorded through an audio recorder and the audio files were later analyzed by a native Spanish speaker in the laboratory. Memory for each sequence was quantified by the number of pictures (excluding the cue) correctly recalled. 


\section{Sequence order recognition analysis}

For Experiment 2, the temporal order memory for picture sequences was later compared to the true order of the sequence, and the result for each trial was coded as the maximum number of pictures (including the cue) correctly ordered in a row.

\section{EEG data analysis}

For each participant, we first used EEG data of the encoding phase to extract epochs for each item within the sequence, namely an EEG epoch for the $1^{\text {st }}, 2^{\text {nd }}, 3^{\text {rd }}, 4^{\text {th }}, 5^{\text {th }}$ and $6^{\text {th }}$ picture. Each epoch had a duration of $2500 \mathrm{~ms}$ and was baseline corrected to the pre-stimulus interval (-100 to $0 \mathrm{~ms})$. We then extracted epochs for the post-episode offset signals after each sequence with duration of $4000 \mathrm{~ms}$ and baseline corrected to the time interval (-100 to $0 \mathrm{~ms})$ before its onset. Finally, we repeated the procedure to extract epochs for the post-item offset of $1500 \mathrm{~ms}$ (with baseline corrected to -100 to $0 \mathrm{~ms}$ ), that corresponded to the inter-stimulus interval separating each item presentation during episodic sequence encoding phase.

\section{Time-resolved Representational Similarity Analysis (RSA)}

For RSA, each EEG epoch data was smoothed by averaging data via a moving window of $100 \mathrm{~ms}$ throughout the EEG epoch (excluding the baseline period) and then down-sampled by a factor of 5 . RSA was performed at individual level and included spatial features (i.e., scalp voltages from all the 28 electrodes) (Silva et al., 2019). The similarity analysis was calculated using Pearson correlation coefficients, which are insensitive to the absolute amplitude and variance of the EEG response.

For both Experiment $1 \&$ 2, we conducted a trial-based RSA between the EEG signal elicited by each encoding item $\left(1^{\text {st }}, 2^{\text {nd }}, 3^{\text {rd }}, 4^{\text {th }}, 5^{\text {th }}\right.$ and $\left.6^{\text {th }}\right)$ and the EEG signal elicited during immediate post-episode offset. After data smoothing and down-sampling, EEG epoch data for each item encoding contained 250 time points (given the $500 \mathrm{~Hz}$ EEG recording sampling rate) covering the $2500 \mathrm{~ms}$ of item picture presentation, and EEG data for each post-episode offset contained 400 time points, equivalent to $4000 \mathrm{~ms}$. Point-to-point correlation values were then calculated, resulting in a single trial 2D similarity matrix with the size of $250 \times 400$, where the $x$ axis represented the offset time points and the $y$-axis represented the encoding time points. $A$ trial-based RSA was computed between EEG patterns elicited by the encoding of $1^{\text {st }}$ to $4^{\text {th }}$ and $1^{\text {st }}$ to $5^{\text {th }}$ picture and the EEG patterns elicited at the immediate post- $4^{\text {th }}$ and post- $5^{\text {th }}$ picture ISI interval (i.e., 1500ms), respectively. More concretely, we conducted RSA between EEG signal elicited at post-stimulus period after the $4^{\text {th }}$ item and EEG patterns triggered during the encoding of each of the preceding $1^{\text {st }}, 2^{\text {nd }}, 3^{\text {rd }}$ and $4^{\text {th }}$ items. Then we repeated the same RSA but between EEG signal elicited at post-stimulus period after the $5^{\text {th }}$ item and EEG patterns triggered during the encoding of each of the preceding $1^{\text {st }}, 2^{\text {nd }}, 3^{\text {rd }}, 4^{\text {th }}$ and $5^{\text {th }}$ items. This 
resulted in 9 similarity matrices in total for each participant. The resulting similarity matrices were then averaged which resulted in a single 2D matrix with size of $250 \times 150$ (i.e., $2500 \mathrm{~ms}$ of picture encoding $\times 1500 \mathrm{~ms}$ of post-stimulus offset or ISI), depicting the overall degree of similarity between EEG patterns elicited during item encoding and post-item offset.

\section{Nonparametric Cluster-Based Permutation Test}

To account for RSA differences between conditions, we employed a nonparametric statistical method (Maris \& Oostenveld, 2007), which identifies clusters of significant points on the resulting $2 \mathrm{D}$ similarity matrix and corrects for multiple comparison based on cluster-level randomization testing to control the family-wise error rate. Statistics were computed on values between conditions for each time point, and based on adjacency in the 2D matrix, adjacent points that passed the significance threshold $(p<0.05$, two-tailed) were selected and grouped together as a cluster. The cluster-level statistics were then calculated by summing up the statistics of all time points within in each identified cluster. The procedure was then repeated 1000 times with randomly shuffled labels across conditions to simulate the null hypothesis. For each permutation, the cluster-level statistics with highest absolute value was registered to construct a distribution of the cluster-level statistics under the null hypothesis. The nonparametric statistical test was calculated by the proportion of permuted test statistics that exceeded the true observed cluster-level statistics.

\section{Linear-mixed effect model}

To investigate the relationship between EEG similarity values and behavioral memory on a trial basis we implemented a Linear Mixed Effect Model (LMM), which accounts for intraand inter-individual variances. We specified in our LMM the correlation values for one specific point on the resulting 2D similarity matrix as the dependent variable and included the following factors as fixed effect variables: the number of items correctly recalled (which ranged from 0 to 5 , without counting the picture cue present at the recall task); the index of an item's order in each sequence $\left(1^{\text {st }}, 2^{\text {nd }}, 3^{\text {rd }}, 4^{\text {th }}, 5^{\text {th }}\right.$ and $\left.6^{\text {th }}\right)$, and the coherence rating provided by the participant to each sequence at encoding (which ranged from 1 to 4 ). Participant number was introduced into the model as the grouping variable, with random intercept and a fixed slope for each of the fixed-effect variables. To balance the requirement for computational power and signal to noise ratio, we further smoothed the resulting $2 \mathrm{D}$ similarity matrix for each item-offset pair by averaging over a moving window of $200 \mathrm{~ms}$ and then down-sampled by the factor of 5 , both smoothing and down-sampling were conducted 2-dimensionally across the $x$ and $y$ axes. We applied the model fitting analysis independently for each position on the resulting $2 \mathrm{D}$ similarity matrix $(50 \times 80)$, then returned the $2 D$ statistics map of the same size for each fixedeffect variable. Here to control for multi-comparison problem, the nonparametric cluster-based permutation test cannot be applied because each permutation represents a sample from the 
null distribution, which is not the case in LMM where it contains a covariance structure induced by multiple levels of relatedness among the individuals. Therefore, we implemented a Bonferroni correction to the statistical threshold to correct for the multiple comparison problem in the resulting statistical maps for each fixed-effect variable. Thus, the resulting statistical map was thresholded with an adjusted alpha level of $\alpha=1.25 \times 10^{-5}(0.05 / 4000)$.

\section{Results}

\section{Experiment 1: Meaningful episodic sequence encoding}

\section{Free recall for meaningful episodes}

In Experiment 1, participants were able to recall on average 2.32 items (SD $=0.456)$ in each series out of the total possible 5 items included in episodic sequence. The mean percentage of trials across participants that successfully recalled $0,1,2,3,4$ and 5 items after the retrieval cue were respectively $24.89 \%$ (SD $=9.34 \%$ ), $8.65 \%(S D=3.41 \%), 14.14 \%$ (SD $=4.94 \%), 24 \%(S D=5.68 \%), 18.91 \%(S D=6.49 \%)$ and $9.41 \%(S D=6.35 \%)$. A repeatedmeasures ANOVA revealed that participants' memory recall differed as a function of number of items recalled following the retrieval cue $\left(F_{(5,120)}=26.227, \mathrm{p}<0.001\right)$ (Fig. 2a). To increase the signal to noise ratio, for later RSA on EEG data, we first adopted a median split approach to separate the trials based on the corresponding task performance. Sequences with 2 or fewer items recalled during the retrieval phase were labeled as Low memory trials and sequences with equal or more than 3 items recalled were labeled as High memory trials. The threshold was selected by its relatively well-balanced separation for number of trials at subject level, as the average percentage of trials after the median split separation was respectively $47.64 \%(S D=12.51 \%)$ for Low memory condition and $52.36 \%(S D=12.51 \%)$ for High memory condition (Wilcoxon signed-rank test: $z=0.821, p=0.412$ ) (Fig. 2b).

\section{Subjective ratings of episodic coherence}

The coherence rating provided a subjective measure of the degree of perceived narrative of each sequence. Due to technical issues, data for coherence ratings of 4 participants were not registered and they could not be included in the analysis. On average, for the remaining 21 participants, sequences were rated as $2.6(S D=0.41)$ (on a scale that ranged from 1 to 4 ), and the mean percentage of trials rated as $1,2,3$, and 4 were respectively $15.34 \%(S D=13.63 \%), 26.79 \%(S D=11.67 \%), 40.36 \%(S D=17.04 \%)$ and $17.51 \%(S D=$ $14.58 \%)\left(F_{(3,60)}=9.889, p<0.001\right)$ (Fig. 2c). After median splitting the trials based on verbal recall performance, trials with High memory showed significantly higher coherence ratings (mean $=2.79, \mathrm{SD}=0.45)$ compared to trials with Low memory (mean $=2.40, \mathrm{SD}=0.45$; paired Student $t$-test: $t_{(20)}=6.166, p<0.001$, two-tailed) (Fig. $2 d$ ). 
a

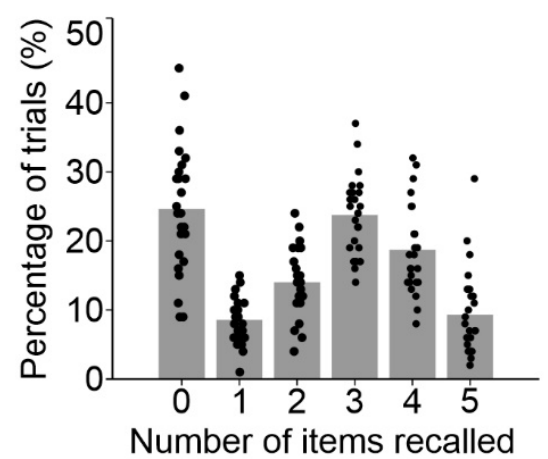

C

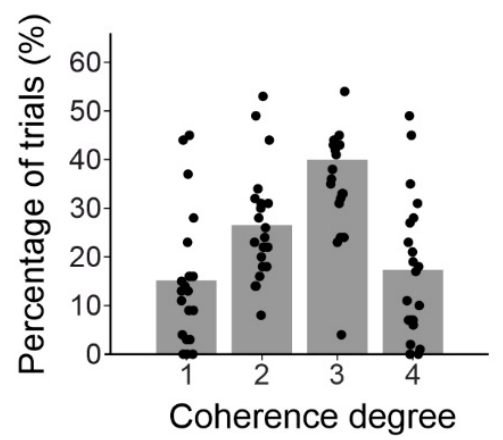

b

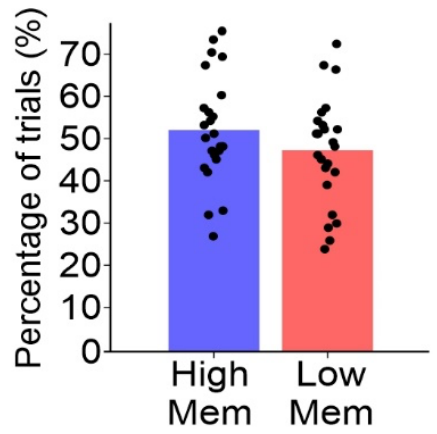

d

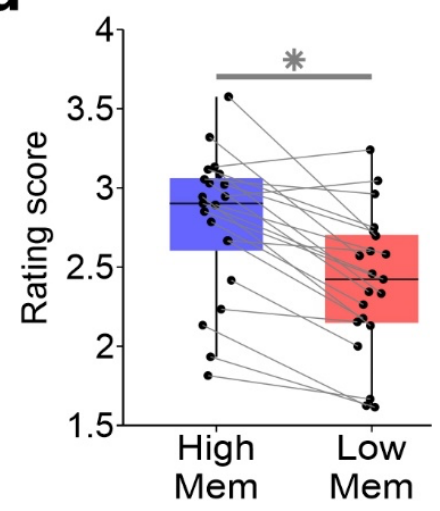

Figure 2. Behavioral results for Experiment 1. a, Percentage of trials at the cued recall task as a function of number of items recalled in each sequence. $\boldsymbol{b}$, Percentage of trials included in the High (High Mem) and Low memory (Low Mem) condition in experiment 1 after implementing the median-split approach. Series with at least 3 item pictures correctly retrieved after cue were counted as High memory trials, otherwise the series were counted as Low memory trials. c , Percentage of trials as a function of the participants' degree of subjective coherence rating. $\boldsymbol{d}$, Mean coherence rating score for trials included in the High and Low memory condition. separated by median-split verbal recall memory. In (a-c), bars represent the average across participants. Each black dot represents values for an individual participant. For all boxplots in (d), the central mark is the median, the edges of the box are the 25 th and 75 th percentiles. ${ }^{*} p<0.05$.

\section{RSA between item sequence and episodic offset at encoding}

We first asked whether EEG patterns induced at the post-episode offset period correlated to EEG patterns elicited by the just encoded picture items within the episodic sequence, and if so, whether the magnitude of such correlation was associated to memory recall at the test. To address this issue, we implemented a trial-based RSA between EEG data elicited at picture item encoding $\left(1^{\text {st }}, 2^{\text {nd }}, 3^{\text {rd }}, 4^{\text {th }}, 5^{\text {th }}\right.$ and $\left.6^{\text {th }}\right)$ with EEG data at the immediately following episode offset period. For each participant, the resulting trial-based RSA values were averaged separately according to two memory conditions: those associated with the participants ability to recall picture items with High memory ( 3 or more items) and those with Low memory (less than 3 items). To account for enough number of EEG trials to be included in both conditions and to ensure these trials included all items (1-6) and post-episode offset 
EEG signal cleaned of artifacts, participants with a low number of trials $(<15 \%)$ in either condition were excluded, resulting in 15 participants for the current analysis.

In both High and Low memory conditions, the results of this analysis revealed an increase in similarity between EEG patterns induced $\sim 400 \mathrm{~ms}-800 \mathrm{~ms}$ at the post-episode offset period and EEG signal elicited $\sim 400 \mathrm{~ms}-1300 \mathrm{~ms}$ at picture item sequence encoding period (Fig. 3a). However, the nonparametric cluster-based permutation analysis identified one statistically significant cluster where similarity values were higher in the High than in the Low memory trials $(p=0.001$; mean $\mathrm{t}$-value $=3.242$, peak $\mathrm{t}$-value $=5.473$ ) (Fig. $3 b)$. We next evaluated whether the similarity between item encoding and post-episode offset period was driven by EEG patterns elicited by specific picture items within the just encoded sequence. We extracted the mean similarity values within the identified cluster for each item-offset pair and computed a repeated-measures ANOVA with two factors: trial condition (High vs Low memory) and encoding item $\left(1^{\text {st }}, 2^{\text {nd }}, 3^{\text {rd }}, 4^{\text {th }}, 5^{\text {th }}\right.$ and $\left.6^{\text {th }}\right)$. The results of this analysis showed a significant main effects for both trial condition $\left(F_{[1,14]}=15.407, p=0.002\right)$ and encoding item $\left(F_{[5,70]}=2.677, p=0.028\right.$; Quadratic trend: $\left.F_{[5,70]}=13.112, p=0.003\right)$, but no significant interaction $\left(F_{[5,70]}=0.315, p=0.902\right)$. (Fig. $\left.3 c\right)$, indicating that episodic offset increase in similarity was not driven by EEG patterns elicited by specific items from the encoded item sequence.

Having shown that neural similarity increases of the just encoded sequence elements was elicited at episodic offset and that it was functionally associated to later memory recall, we then leveraged this to explore the relationship between the magnitude of memory reinstatement and the numbers of items to be recalled correctly later. At the same time, we asked whether the observed effects could be simply explained by the participants' subjective feeling of coherence of the episode, as we found that subjective ratings of coherence were higher for High than for Low memory condition. To address these issues, we applied a LMM to our trial-based RSA data (see Methods). Two participants from the previous RSA analysis were not included here due to the missing data for coherence rating, resulting in 13 participants in total for the LMM analysis. The result revealed one time interval that survived the statistical threshold for the fixed effect variable total number of items correctly recalled (one-tailed, mean $t$-value $=5.032$, peak $t$-value $=5.621, p<1.25 \times 10^{-5}$, Bonferroni corrected) (Fig. 3d). The time interval, which covered $\sim 300 \mathrm{~ms}-700 \mathrm{~ms}$ of post-episode offset and $\sim 300 \mathrm{~ms}-900 \mathrm{~ms}$ of item encoding, indicated the region where the degree of neural similarity of each item elicited during post-episode offset was significantly positively correlated with total number of items to be recalled in the corresponding sequence. However, no significant point exceeding the statistical threshold was identified on the statistical map accounting for the variable indexing order position in sequence nor for the variable indexing coherence rating. 

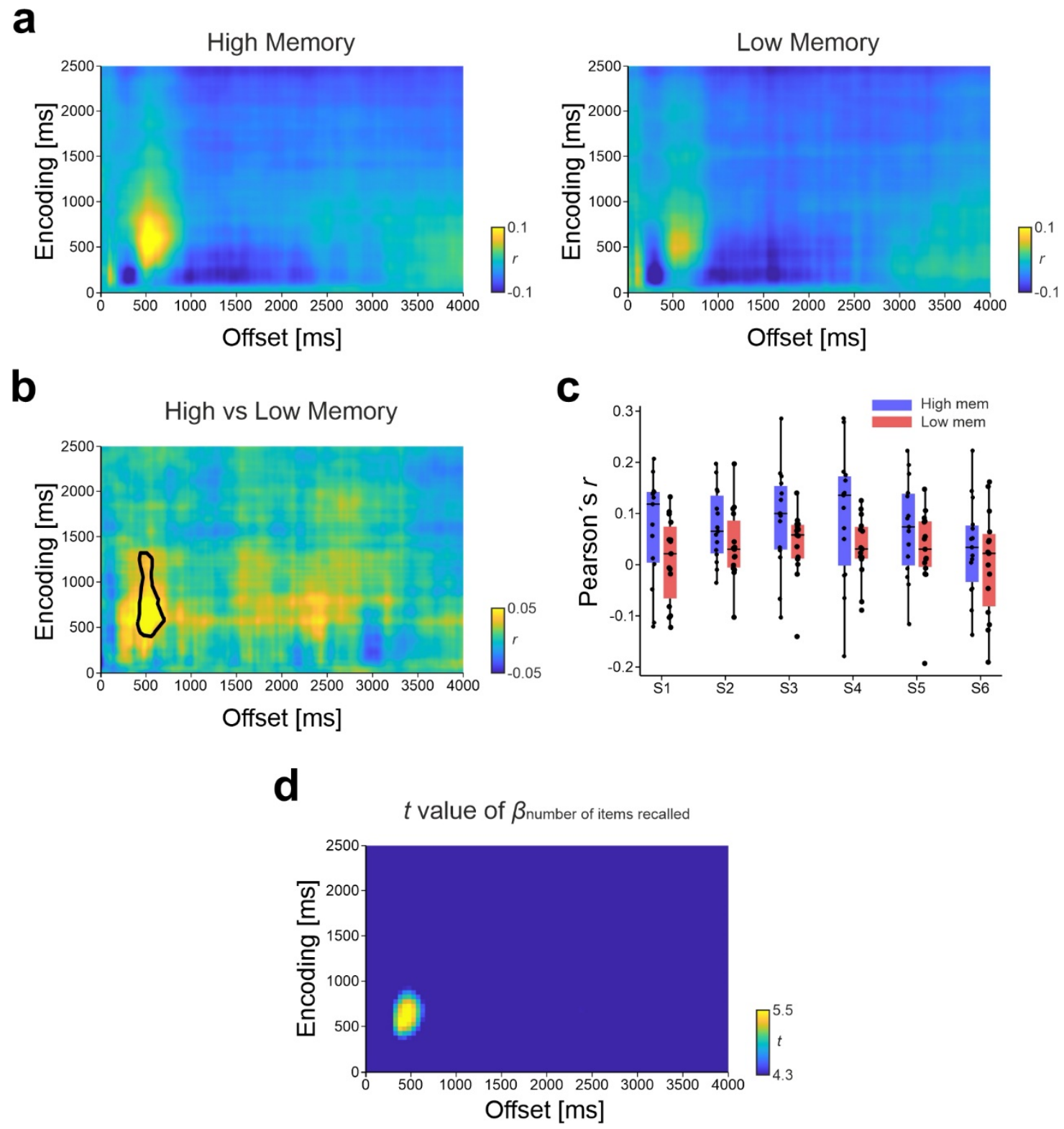

Figure 3. Neural similarity results at post-encoding period for Experiment 1. a, Time-resolved degree of neural similarity between item picture encoding and post-encoding offset for events with High or Low memory at test. $\boldsymbol{b}$, Difference between similarity values for the two conditions. Statistically significant $(p<0.05$, clusterbased permutation test) higher similarity value $\sim 400 \mathrm{~ms}-800 \mathrm{~ms}$ at the post-episode offset period with $\sim 400 \mathrm{~ms}$ - 1300ms at item picture encoding period was found for events with higher recall performance (indicated by a black thick line). $c$, In all boxplots the central mark is the median across participants, the edges of the box are the 25th and 75th percentiles. They depict the degree of similarity within the identified cluster for each item encoding with its corresponding offset period. Each black dot represents values for an individual participant. High memory sequence showed significantly greater similarity across encoding items. $\boldsymbol{d}$, $t$-value map of the variable numbers of item recalled reveals the area that exceeded the significance threshold after Bonferroni correction with adjusted alpha level of $\alpha=1.25 \times 10^{-5}$ (one-tailed). No area exceeding the significance threshold was found for $t$-value map of the variable serial position of item in sequence neither for that of the variable coherence rating.

\section{RSA between item encoding and sequence item immediate offset}

Next, we asked whether the increases in neural similarity between EEG patterns elicited at item sequence encoding and encoding offset were specific to post-episode period, 
or alternatively, whether they could be also found at offset periods immediately following picture encoding. To address this issue, we implemented the RSA between EEG pattern elicited by each item in the encoding sequence and the EEG signal pattern induced during the immediate post-item offset period. The current analysis was centered in the post-item offset period after the $4^{\text {th }}$ and $5^{\text {th }}$ item as this represented a delay period, as in episodic offset period, that is preceded by the encoding of multiple items from a sequence but differed in that the encoding of episode is not completed yet. At the same time, this research strategy allowed us to implement the same median split analysis used in our previous analysis (i.e., whether or not at least 3 items after the picture cue were correctly remembered), thereby enabling the comparison of the RSA results from the two conditions later on. 8 out of the total 25 participants were excluded for this analysis due to insufficient number of clean EEG trials in either conditions (i.e., at least $15 \%$ of total number of trials in either encoding item of either condition). The result of this analysis showed no clear increases in neural similarity in either High or Low memory conditions (Fig. 4a) and that no cluster of similarity values were accounted when the two conditions were compared with a cluster-based permutation test (Fig. $4 b$ ). With the aim to further examine that the increase in neural similarity was specific to the episodic offset period, we directly compared the neural similarity findings at the first $1500 \mathrm{~ms}$ of the episodic offset vs at the $1500 \mathrm{~ms}$ of the $4^{\text {th }}$ and $5^{\text {th }}$ post-item offset. The results of this analysis confirmed a cluster of significantly higher neural similarity at the episodic offset condition (Fig. 4C), thereby corroborating the notion that the increase in neural similarity was specific to post-episodic encoding delay period.

a

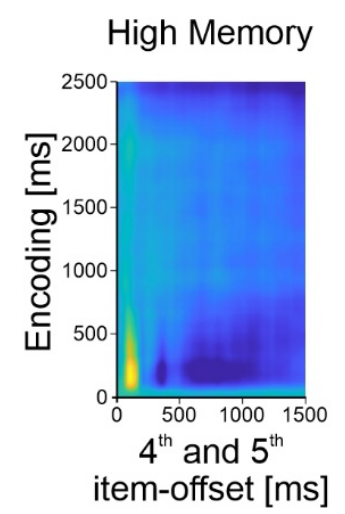

Low Memory

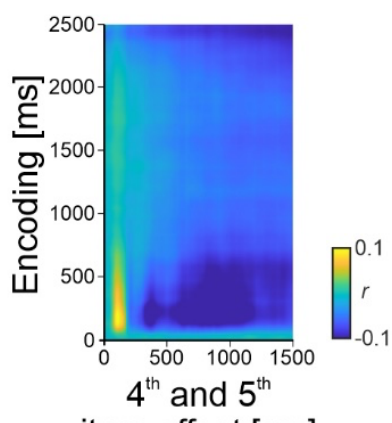

item-offset [ms] b

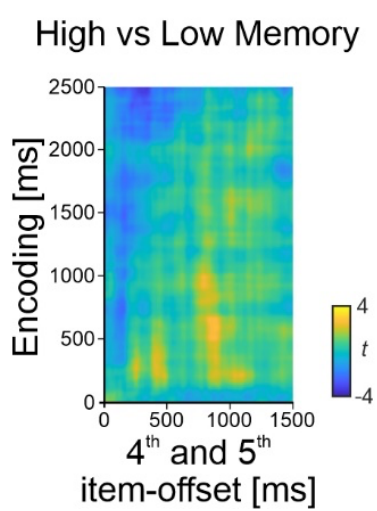

C

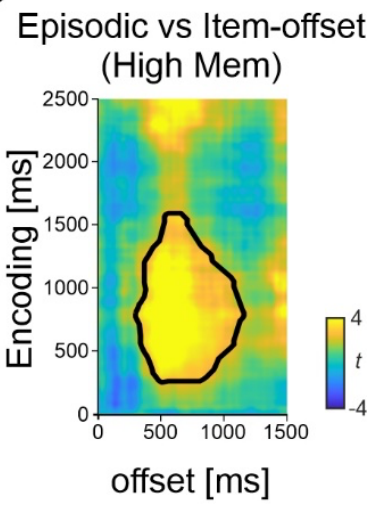

Figure 4. $a$, Time-resolved degree of similarity between item picture encoding and $4^{\text {th }}$ and $5^{\text {th }}$ post-item following the events that were later recalled with High or Low memory. $\boldsymbol{b}$, Difference (expressed in $t$ values, uncorrected) between similarity values for the $4^{\text {th }}$ and $5^{\text {th }}$ post-item High and Low memory conditions. No cluster indicated significantly different similarity values between conditions (two-tailed, $p<0.05$, cluster-based permutation test). $c$, Difference (expressed in $t$ values, uncorrected) between similarity values for episodic offset and the $4^{\text {th }}$ and $5^{\text {th }}$ post-item offset in the High memory condition. Higher neural similarity was found during the episodic offset compared to item-offset. (the significant cluster is indicated by a black thick line. $p<0.05$, corrected with a cluster-based permutation test). 
bioRxiv preprint doi: https://doi.org/10.1101/2021.04.13.439658; this version posted April 14, 2021. The copyright holder for this preprint (which was not certified by peer review) is the author/funder, who has granted bioRxiv a license to display the preprint in perpetuity. It is made available under aCC-BY-NC 4.0 International license.

\section{Experiment 2: Non-meaningful episodic sequence encoding}

\section{Behavioral results}

In general, participants were able to recall on average 0.14 items $(S D=0.176)$ out of the possible five (picture cue was not included in the counting) in each series. The mean percentage of trials across participants to successfully recall $0,1,2,3,4$ and 5 items after the cue were respectively $89.23 \%(S D=11.92 \%), 7.98 \%(S D=8.27 \%), 1.96 \%(S D=3.14 \%)$, $0.77 \%(\mathrm{SD}=1.84 \%), 0.06 \%(\mathrm{SD}=0.32 \%)$ and $0 \%(\mathrm{SD}=0 \%)\left(F_{[5,135]}=795.913, p<0.001\right)$ (Fig. 5a). Even though participants were unable to verbally recall almost any item from an encoded sequence, they showed above chance performance in the order item sequence recognition task.

a

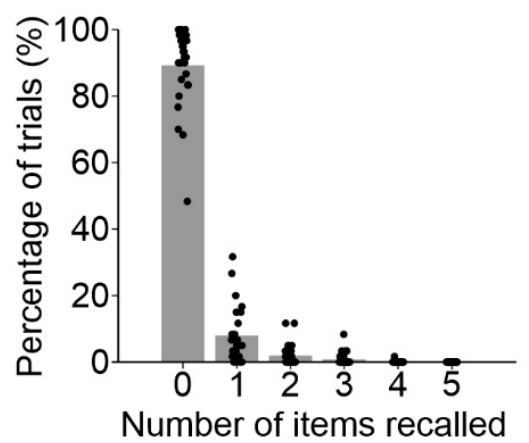

C

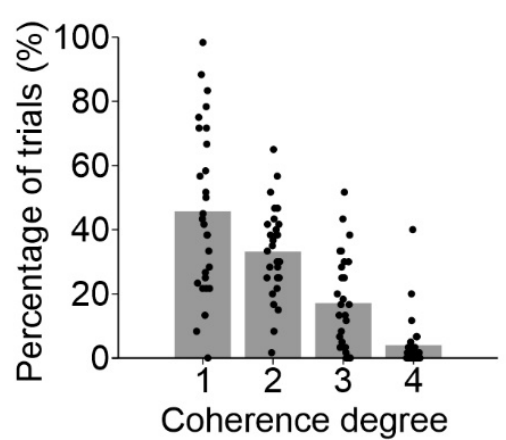

b

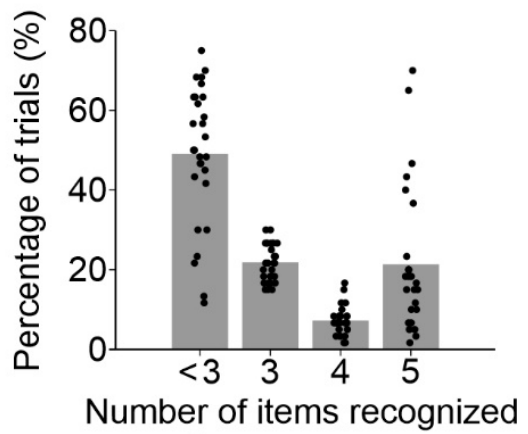

in a sequence

d

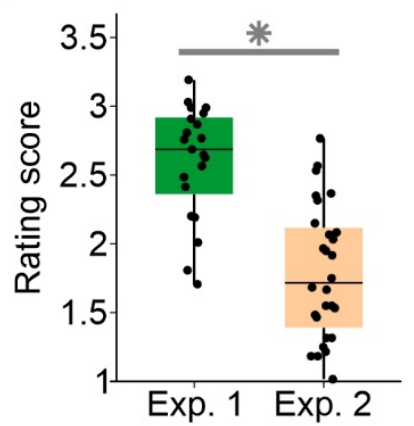

Figure 5. Behavioral results for Experiment 2 a, Percentage of trials separated by number of pictures correctly recalled after the picture. $\boldsymbol{b}$, A temporal order sequence recognition memory test was added after the cued recall task. In the recognition memory task, all 6 pictures from the sequence were presented in random positions at the screen, including the cue, and participants had $30 \mathrm{~s}$ to order the correct temporal structure of the encoded sequences. The figure showed percentage of trials separated by the sequence recognition score. The score is quantified as the maximum number of pictures correctly ordered consecutively in one trial. A trial with less than 2 correct pictures consecutively ordered following either the cue, the $2^{\text {nd }}$, the $3^{\text {rd }}$ or the $4^{\text {th }}$ picture (score less than 3) was counted as no sequence recognized. c, Percentage of trials separated by degree of coherence rating. $\boldsymbol{d}$, Mean coherence rating score of sequences in Experiment 1 vs Experiment 2. In (a-c), bars represent the average across participants. Each black dot represents values for an individual participant. For all boxplots in (d), the central mark is the median, the edges of the box are the 25th and 75th percentiles. ${ }^{*} p<0.05$. 
We analyzed these data by quantifying the maximum number of pictures correctly ordered consecutively for each given trial sequence. A trial was considered to have been recognized if the participant reported at least 3 items in correct order from the sequence. On average, the percentage of trials to have scored less than 3, 3, 4 and 5 (i.e., all pictures in order following the cue) were respectively $49.11 \%$ (SD = 17.31\%), $21.85 \%(S D=4.72 \%), 7.26 \%$ $(\mathrm{SD}=3.55 \%)$ and $21.79 \%(\mathrm{SD}=17.62 \%)\left(F_{(3,81)}=39.777, p<0.001\right)($ Fig. $5 b)$. The participants provided correct order recognition for $50.89 \%(S D=17.31 \%)$ of the trials, which is statistically significantly above chance (chance level $=15 \%, t_{(27)}=10.975 ; p<0.001$, twotailed). Finally, the average coherence rating for sequences in Experiment 2 was 1.79 (SD = 0.48 ), and the mean percentage of trials rated as $1,2,3$, and 4 were respectively $45.71 \%$ (SD $=25.96 \%), 33.15 \%(\mathrm{SD}=14.20 \%), 17.14 \%(\mathrm{SD}=14.68 \%)$ and $3.99 \%(\mathrm{SD}=8.31 \%)\left(F_{(3,81)}=\right.$ 24.108, $p<0.001$ ) (Fig. 5c). In general, sequences in Experiment 1 were rated significantly higher than sequences in Experiment $2\left(t_{(47)}=6.164, p<0.001\right.$, two-tailed) (Fig. $5 d$ ), which suggested that the subjective feeling of coherence matched the general manipulation of the experiment.

\section{$R S A$ between item sequence and episodic offset at encoding}

As in experiment 1, we computed a trial-based RSA between 2500ms EEG patterns elicited by each sequence picture item and the corresponding EEG data induced at the 4000 ms episodic offset. The parameters for data smoothing and down sampling was kept the same as Experiment 1. For each participant, the resulting 2D similarity matrix was first averaged within each item-offset pair and then across pairs. 10 out of the total 28 participants were excluded for this analysis due to insufficient number of clean trials for all items (at least $15 \%$ of total number of trials in each item-offset pair). Thus, a total of 18 participants were included in the analysis. However, and contrary to as in experiment 1, the result of RSA for Experiment 2 did not show any observable neural similarity increase at the offset period (Fig. $6 a)$. To assess the extent to which neural similarity patterns seen in experiment 1 differed from those obtained in experiment 2 , we separately compared the neural similarity values for High and for Low memory conditions in Experiment 1 with those obtained in Experiment 2. The results of these analyses revealed that neural similarity increase found at early offset period in experiment 1 , for both High and Low memory conditions, was statistically different from similarity values at the offset period in experiment 2 (Fig. 6b). More concretely, this analysis returned one significant cluster $(p=0.022$ (corrected), mean $t$-value $=3.354$, peak $t$-value $=$ 5.059 ), that comprised higher neural similarity values for EEG data within $\sim 400-1000 \mathrm{~ms}$ time range from post-episode offset period and $\sim 200-1400$ ms time range from item picture encoding from High memory trials in experiment 1 over trials from experiment 2. A similar cluster in its timing ( $450-1000$ ms of post-episode offset and $\sim 200-1100$ ms of item encoding) 
with significantly higher neural similarity values was found when comparing Low memory trials from experiment 1 with trials from experiment 2 ( $p=0.026$ (corrected), mean $t$-value $=2.928$, peak $t$-value $=4.237)$.

a

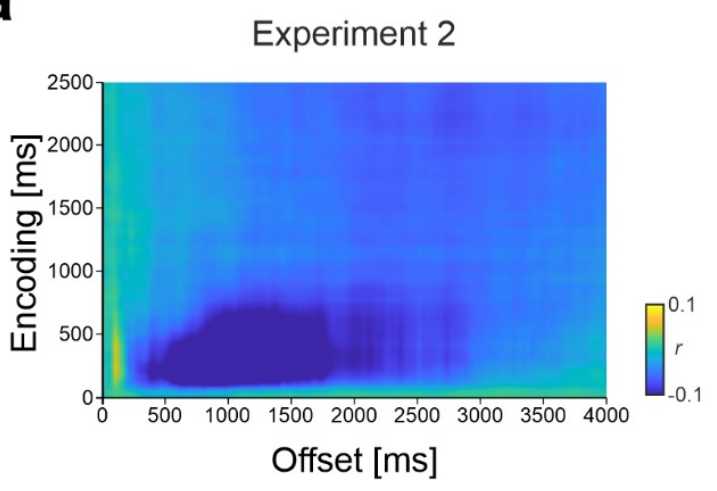

b

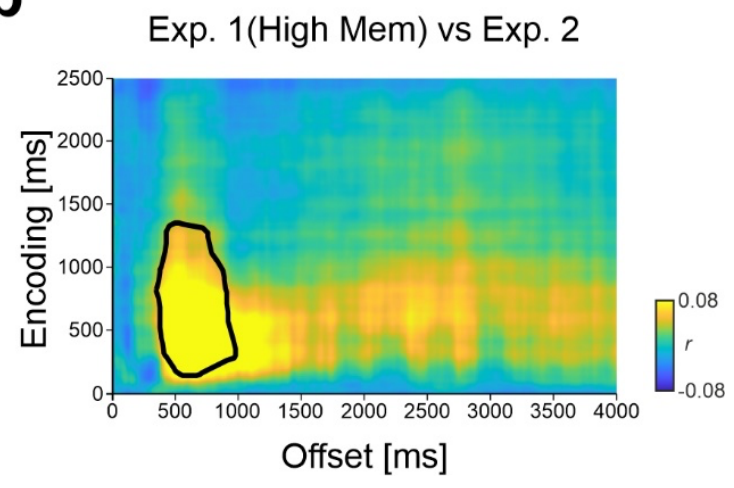

Figure 6. Neural similarity in experiment 2. a, Neural similarity between item picture encoding at postencoding period for Experiment 2. $\boldsymbol{b}$, Higher neural similarity was found during the offset period in High memory trials in experiment 1 when compared to the offset period in in experiment 2 (the significant cluster is indicated by a black thick line. $p<0.05$, corrected with a cluster-based permutation test).

\section{Discussion}

Here, we investigated in healthy human participants whether the reinstatement of a just encoded episode is a mechanism selectively engaged to support rapid memory formation of single episodes. We used representational similarity analysis of scalp electroencephalography recordings during the encoding of sequences of pictures depicting unique episodic-like events and found that EEG patterns elicited during picture viewing correlated with EEG patterns at the episode offset. The degree of episodic offset-reactivation predicted memory recollection of the encoded picture sequence and it was not present when participants encoded sequences of pictures that were not perceived as meaningful episodes. These results indicate that episodic offset memory reinstatement is a mechanism selectively engaged to support rapid memory formation of single episodes induced once participants perceive a meaningful episode to be complete.

Why would post-encoding neural reactivation be important for long-term memory formation for first-time experienced sequential episodes? Studies on sequential learning have demonstrated that memory encoding is enhanced for information presented at event boundaries, when shifts in stimulus category or object location occur, and that these memory enhancements are related to neural activity in the hippocampus and prefrontal cortex (e.g., DuBrow and Davachi, 2016; Heusser et al, 2016; Horner et al., 2016). fMRI research using movie clips as encoded material show that the hippocampus becomes more active at event boundaries during memory encoding and this hippocampal activity is predictive of memory 
retrieval (Ben-Yakov et al., 2013, 2018; Baldassano et al., 2017). In line with these findings, we recently showed that event boundaries, operationalized as transition points in the encoding time whereby one episode ends and new one starts, triggered the memory reinstatement of the just encoded event information and that the degree of boundary-elicited memory reactivation was associated to memory performance in a subsequent test (Sols et al., 2017; Silva et al., 2019). However, current results extend previous findings in several ways.

First, we found that memory reinstatement occurs only at the offset of a sequential event episode. Importantly, and in contrast to our previous findings, memory reinstatement was not elicited by detected transitions between the encoding of two consecutive episodes but by moments in time whereby individuals perceived the encoding of an event to be concluded. We argue that memory reinstatement findings in the current experiment describe an endogenous process that characterizes the need to wait for the optimal moment to form a memory representation that integrates a complete and coherent structure of the unfolding event experience into long-term memory. Together with our previous findings, this raises the interesting possibility that rapid event-offset memory reinstatement had at list two different functional properties: to bridge temporally adjacent events during continuous encoding (i.e., Sols et al., 2017) and to integrate a just encoded event into a coherent long-term memory trace (i.e., current results). This dissociated role of event-offset memory reinstatement is also supported from the data of our second experiment, whereby offset periods following the encoding of a non-meaningful sequence of pictures did not elicit any observable memory reactivation. These results are be in line with the hypothesis that post-encoding memory reinstatement require the engagement of external (e.g., driven by contextual shifts in stimuli properties between two consecutive episodes as in Sols et al., 2017) and/or endogenously driven processes (as in the current study).

Our findings that memory reinstatement was only evident at the episodic offset but not at offset periods between pictures depicted during sequential encoding supports the notion that memory reinstatement does not merely reflect an ongoing mechanism that links items associated during encoding. Instead, it suggests that it contributes to the integration of event components into long-term memory once an individual perceives a meaningful episode is concluded. Previous fMRI research has highlighted that the primary role of hippocampal offset signal in reflecting binding operations of stimuli that just co-occurred within the same spatialtemporal context (Staresina and Davachi, 2009; Ritchey and Cooper, 2020). Similarly, fMRI studies (Baldassano et al., 2017) and electroencephalographic recordings from implanted electrodes in epileptic patients (Michelmann et al., 2020) revealed that the degree to which offset hippocampal activity couples with cortical patterns of activity during a continuous stream of stimuli predicts pattern reinstatement during later recall, thereby indicating that the 
hippocampus may be responsible for binding cortical representations into a memory trace online during encoding (McClelland et al., 1995; Norman and O’Reilly, 2003; Moscovitch et al., 2005).

The fact that memory reactivation was found during a delay period immediately following encoding can be seen as a reflection of a mechanism inherently linked to the working memory (WM) maintenance of the encoded sequence of pictures. However, several observations in our results suggest the reinstatement at the episode offset cannot be explained solely by WM processes. First, post-encoding memory reinstatement predicted participants' ability to recollect the episodic picture content in a later test but not their subjective rating of coherence that immediately followed the delay period. Should post-encoding memory reinstatement be a mechanism supporting WM maintenance we would expect it to be at least partially associated with the individual's ability to evaluate the episodic coherence of the encoded picture sequence after the delay period. Second, post-encoding memory reinstatement in our study was circumscribed to the beginning but not throughout the postencoding delay period. However, studies investigating the neural substrates of WM have shown that delay maintenance is associated to a sustained increase in activation of neocortical structures (Fuster and Alexander, 1971). Should post-encoding memory reinstatement be associated to the sustained increase neural activity during the delay we would expect it to be observed over extended portions of the offset delay period and not only at the beginning of it. More recently, it has been argued that such above-threshold delay-period activity may support functions other than information storage per se (D'Esposito and Postle, 2015) and the existence of other neural coding mechanisms such as "activity-silent" states (Strokes, 2015) and dynamic coding schemes (Liu et al., 2020). However, these neural representational formats are still susceptible to be identified with the implementation of multivariate decoding approaches, such as the one implemented in the current design, thereby rendering unlikely they were unobservable throughout the delay period in our study. Finally, the results of our second experiment, where participants were unable to recollect the encoded event episodes, but they could reliably provide a subjective rating for each picture sequence after the delay period, showed an absence of memory reinstatement at the offset period. Again, though the statistical absence of an effect cannot guarantee the absence of the effect, these results add further support that notion that post-encoding memory reinstatement reflects encoding to longterm memory, rather than working memory maintenance.

Single-cell recordings from the rodent hippocampus during navigational tasks have shown that neural replay can be even observed after the first lap on a novel track (Foster and Wilson, 2006), thereby suggesting that neural replay after single-shot learning may reflect early stages encoding recent experiences in long-term memory. More strikingly, rodent 
research has shown that post-encoding replay may preserve the temporal structure of the encoded event sequence in a compressed time-manner (Csicsvari et al., 2007; Diba \& Buzsaki 2007; Foster \& Wilson 2006; Gupta et al., 2010; Karlsson and Frank, 2009). Our findings on the basis of scalp EEG recordings are blind to whether memory reinstatement at episodic offset period relies on memory replay of a temporally preserved structure of the encoded sequence but they showed that the reinstatement of the encoded sequence was limited to a very short time window ( $400 \mathrm{~ms})$ at the episode offset. Future studies using brain acquisition approaches more sensitive to hippocampal activity, such as MEG (e.g., Liu et al., 2019) or intracortical recordings directly from the human hippocampus (e.g., Vaz et al., 2020) may help disambiguate whether the compressed episodic offset memory reinstatement preserves a temporal structure of an encoded sequence episode.

To conclude, we have shown that episodic offset memory reinstatement is selectively engaged to support successful encoding of sequential picture series with a narrative structure. These results shed light on the neural mechanisms that support the rapid learning of novel episodes that unfold over time in humans and how they serve to selectively transform experiences into long-term memory representations that can be later recalled.

\section{References}

Baldassano, C., Chen, J., Zadbood, A., Pillow, J. W., Hasson, U., \& Norman, K. A. (2017). Discovering event structure in continuous narrative perception and memory. Neuron, 95(3), 709-721.

Ben-Yakov, A., Eshel, N., \& Dudai, Y. (2013). Hippocampal immediate poststimulus activity in the encoding of consecutive naturalistic episodes. Journal of Experimental Psychology: General, 142(4), 1255.

Ben-Yakov, A., \& Henson, R. N. (2018). The hippocampal film editor: sensitivity and specificity to event boundaries in continuous experience. Journal of Neuroscience, 38(47), 10057-10068.

Carr, M. F., Jadhav, S. P., \& Frank, L. M. (2011). Hippocampal replay in the awake state: a potential substrate for memory consolidation and retrieval. Nature neuroscience, 14(2), 147.

Csicsvari, J., O'Neill, J., Allen, K., \& Senior, T. (2007). Place-selective firing contributes to the reverse-order reactivation of CA1 pyramidal cells during sharp waves in open-field exploration. European Journal of Neuroscience, 26(3), 704-716.

Davachi, L. (2006). Item, context and relational episodic encoding in humans. Current opinion in neurobiology, 16(6), 693-700.

Diana, R. A., Yonelinas, A. P., \& Ranganath, C. (2007). Imaging recollection and familiarity in the medial temporal lobe: a three-component model. Trends in cognitive sciences, 11(9), 379386. 
Diba, K., \& Buzsáki, G. (2007). Forward and reverse hippocampal place-cell sequences during ripples. Nature neuroscience, 10(10), 1241-1242.

DuBrow, S., \& Davachi, L. (2013). The influence of context boundaries on memory for the sequential order of events. Journal of Experimental Psychology: General, 142(4), 1277.

DuBrow, S., \& Davachi, L. (2014). Temporal memory is shaped by encoding stability and intervening item reactivation. Journal of Neuroscience, 34(42), 13998-14005.

DuBrow, S., \& Davachi, L. (2016). Temporal binding within and across events. Neurobiology of learning and memory, 134, 107-114.

D'Esposito, M., \& Postle, B. R. (2015). The cognitive neuroscience of working memory. Annual review of psychology, 66, 115-142.

Eichenbaum, H., Yonelinas, A. P., \& Ranganath, C. (2007). The medial temporal lobe and recognition memory. Annu. Rev. Neurosci., 30, 123-152.

Ezzyat, Y., \& Davachi, L. (2011). What constitutes an episode in episodic memory?. Psychological science, 22(2), 243-252.

Foster, D. J., \& Wilson, M. A. (2006). Reverse replay of behavioural sequences in hippocampal place cells during the awake state. Nature, 440(7084), 680-683.

Foster, D. J. (2017). Replay comes of age. Annual review of neuroscience, 40, 581-602.

Fuster, J. M., \& Alexander, G. E. (1971). Neuron activity related to short-term memory. Science, 173(3997), 652-654.

Gupta, A. S., van der Meer, M. A., Touretzky, D. S., \& Redish, A. D. (2010). Hippocampal replay is not a simple function of experience. Neuron, 65(5), 695-705.

Heusser, Andrew C., et al. "Episodic sequence memory is supported by a theta-gamma phase code." Nature neuroscience 19.10 (2016): 1374-1380.

Horner, A. J., Bisby, J. A., Wang, A., Bogus, K., \& Burgess, N. (2016). The role of spatial boundaries in shaping long-term event representations. Cognition, 154, 151-164.

Karlsson, M. P., \& Frank, L. M. (2008). Network dynamics underlying the formation of sparse, informative representations in the hippocampus. Journal of Neuroscience, 28(52), 1427114281.

Karlsson, M. P., \& Frank, L. M. (2009). Awake replay of remote experiences in the hippocampus. Nature neuroscience, 12(7), 913-918.

Kurby, C. A., \& Zacks, J. M. (2008). Segmentation in the perception and memory of events. Trends in cognitive sciences, 12(2), 72-79.

Liu, J., Zhang, H., Yu, T., Ni, D., Ren, L., Yang, Q., ... \& Xue, G. (2020). Stable maintenance of multiple representational formats in human visual short-term memory. Proceedings of the National Academy of Sciences, 117(51), 32329-32339.

Liu, Y., Dolan, R. J., Kurth-Nelson, Z., \& Behrens, T. E. (2019). Human replay spontaneously reorganizes experience. Cell, 178(3), 640-652.

Maris, E., and Oostenveld, R. (2007). Nonparametric statistical testing of EEG- and MEG-data. J. Neurosci. Methods 164, 177-190. 
McClelland, J. L., McNaughton, B. L., \& O'Reilly, R. C. (1995). Why there are complementary learning systems in the hippocampus and neocortex: insights from the successes and failures of connectionist models of learning and memory. Psychological review, 102(3), 419.

Michelmann, S., Price, A. R., Aubrey, B., Doyle, W. K., Friedman, D., Dugan, P. C., ... \& Norman, K. A. (2020). Moment-by-moment tracking of naturalistic learning and its underlying hippocampo-cortical interactions. bioRxiv 2020.12.09.416438; doi: https://doi.org/10.1101/2020.12.09.416438.

Moscovitch, M., Rosenbaum, R. S., Gilboa, A., Addis, D. R., Westmacott, R., Grady, C., ... \& Nadel, L. (2005). Functional neuroanatomy of remote episodic, semantic and spatial memory: a unified account based on multiple trace theory. Journal of anatomy, 207(1), 35-66.

Norman, K. A., \& O'Reilly, R. C. (2003). Modeling hippocampal and neocortical contributions to recognition memory: a complementary-learning-systems approach. Psychological review, 110(4), 611.

Paller, K. A., \& Wagner, A. D. (2002). Observing the transformation of experience into memory. Trends in cognitive sciences, 6(2), 93-102.

Radvansky, G. A. (2012). Across the event horizon. Current Directions in Psychological Science, 21(4), 269-272.

Ranganath, C. (2010). A unified framework for the functional organization of the medial temporal lobes and the phenomenology of episodic memory. Hippocampus, 20(11), 12631290.

Ritchey, M., \& Cooper, R. A. (2020). Deconstructing the posterior medial episodic network. Trends in cognitive sciences.

Schuck, N. W., \& Niv, Y. (2019). Sequential replay of nonspatial task states in the human hippocampus. Science, 364(6447).

Silva, M., Baldassano, C., \& Fuentemilla, L. (2019). Rapid memory reactivation at movie event boundaries promotes episodic encoding. Journal of Neuroscience, 39(43), 8538-8548.

Sinclair, A. H., \& Barense, M. D. (2019). Prediction error and memory reactivation: how incomplete reminders drive reconsolidation. Trends in neurosciences, 42(10), 727-739.

Sols, I., DuBrow, S., Davachi, L., \& Fuentemilla, L. (2017). Event boundaries trigger rapid memory reinstatement of the prior events to promote their representation in long-term memory. Current Biology, 27(22), 3499-3504.

Staresina, B. P., \& Davachi, L. (2009). Mind the gap: binding experiences across space and time in the human hippocampus. Neuron, 63(2), 267-276.

Stokes, M. G. (2015). 'Activity-silent'working memory in prefrontal cortex: a dynamic coding framework. Trends in cognitive sciences, 19(7), 394-405.

Tulving, E. (1983). Elements of episodic memory.

Vaz, A. P., Wittig, J. H., Inati, S. K., \& Zaghloul, K. A. (2020). Replay of cortical spiking sequences during human memory retrieval. Science, 367(6482), 1131-1134.

Zacks, J. M., Speer, N. K., Swallow, K. M., Braver, T. S., \& Reynolds, J. R. (2007). Event perception: a mind-brain perspective. Psychological bulletin, 133(2), 273. 